In $\ 1378$ Abs. 2 BGB-E ist vorgesehen, dass die Ausgleichsforderung nicht begrenzt wird, wenn sie wegen fiktiver Hinzurechnung verausgabter Summen die Hälfte des vorhandenen Vermögens übersteigt. Der unredliche Ehegatte muss dann sein gesamtes Vermögen hergeben und sich sogar verschulden, um die Zugewinnausgleichsforderung zu erfüllen. ${ }^{7}$ Der unredliche Ehegatte soll nicht geschützt werden und nicht im Besitz der „verschwundenen“ Habe bleiben.

\ 1390 BGB-E enthält einen Herausgabeanspruch gegen Dritte, die Empfänger der illoyalen Vermögensminderung sind, wenn die Ausgleichsforderung das vorhandene Vermögen des Ehegatten übersteigt; die Voraussetzungen des Herausgabeanspruches zu der bisherigen Bestimmung ändern sich. ${ }^{8}$

Allerdings dürfte auch mit diesen Änderungen nicht die Gefahr beseitigt sein, dass alle illoyalen Vermögensverschiebungen verhindert bzw. ausgeglichen werden. Denn das praktische Problem ist, dass solche Manipulationen häufig schwer nachzuvollziehen und zu beweisen sind. Erwägenswert wäre, eine generelle Auskunftspflicht für die gesamte Dauer der Ehe einzurichten.

Viele Ehepaare haben eine Aufgabenverteilung dergestalt, dass sich der eine um die Finanzen kümmert - oft der Mann - und die Frau keinerlei Interesse und Einblick in diese Materie hat. Das mag gut sein für gute Zeiten; wenn es aber um die Auseinandersetzung geht, dann sollten beide berechtigt sein zu erfahren, was im Laufe der Ehe mit den gemeinsamen Geldern und dem Vermögen passiert ist. Aus dem Grundsatz der ehelichen Solidarität lässt sich ein solcher Anspruch zwanglos herleiten.

Der Entwurf befasst sich nicht damit, dass Wertsteigerungen des Anfangsvermögens oder privilegierten Erwerbs ausgleichspflichtig sind.

Es ist eine Frage der Position, ob man es als gerecht empfindet, dass nach gescheiterter Ehe ein Partner an Vermögenszu- wächsen teilhat, die nichts mit einer gemeinsamen Leistung zu tun haben, die während der Ehe erbracht wurde. Wenn die Eheleute zusammenbleiben, sollen beiden derartige Erträge sicherlich gebühren. Ob jedoch ein Anspruch auf derartige Werte auch einem Ehepartner/einer Ehepartnerin zusteht, wenn er oder sie aus der Ehe ausbricht, ist meines Erachtens nicht unbedingt einzusehen bzw. zumindest diskutabel.

Im Vorfeld unserer Stellungnahme haben wir diskutiert, ob nicht generell der Errungenschaftsgemeinschaft als gesetzlichem Güterstand der Vorzug einzuräumen sei. ${ }^{9}$

Die Zugewinngemeinschaft findet ihresgleichen im europäischen Familienrecht nicht. Der häufigste Güterstand in Europa ist die Errungenschaftsgemeinschaft, die ja auch in Deutschland bis zur Mitte des letzten Jahrhunderts einer der gesetzlichen Güterstände war. Es stellt sich die Frage, ob die Zugewinngemeinschaft als gesetzlicher Güterstand nicht nach wie vor Schwächen aufweist, die die Errungenschaftsgemeinschaft vorzugswürdig erscheinen lassen. Hingewiesen sei auf die vorstehend erwähnten Manipulationsmöglichkeiten oder etwa auf die ausgleichspflichtigen Wertzuwächse, die ohne Leistung des Anspruchsberechtigen bestehen. Auch ist in der Praxis oftmals das System der Zugewinngemeinschaft Laien schwer zu vermitteln; das reine Stichtagsprinzip ohne ausreichende Möglichkeit der Bereinigung zu Ehezeiten erfolgter Vermögensverschiebungen ist Laien vielfach nicht als gerecht darzustellen. Die jetzige Rechtsänderung dürfte innerhalb der europäischen Entwicklung nicht das letzte Wort zum ehelichen Güterrecht gewesen sein.

\footnotetext{
7 Koch, a.a.O.

8 Näheres hierzu vgl. Koch, a.a.O., S. 1129.

9 Vgl. dazu auch, ob die Reformen im Familienrecht Kosmetik oder Kurskorrektur sein sollen, Born in NJW 2008, 2289 ff.
}

\title{
Gesetz zur Strukturreform des Versorgungsausgleichs
}

\section{Der lange Weg zu mehr Gerechtigkeit?}

\section{Stand des Gesetzgebungsverfahrens}

Nachdem sich die vom Bundesministerium der Justiz im Jahr 2003 eingesetzte Kommission in ihrem Abschlussbericht nicht auf eine umfassende Neuordnung und Neubewertung der Versorgungssysteme in allen ihren Facetten verständigen konnte, ist vor dem Hintergrund mahnender Entscheidungen aus Karlsruhe eine Reform unumgänglich. ${ }^{1}$

Der Diskussionsentwurf vom 29. August 2007, der in seinen Ausgleichsformen nahezu unverändert dem Referentenentwurf vom 12. Februar 2008 (und auch dem Regierungsentwurf) entspricht, trifft überwiegend auf Zustimmung.

Bei näherer Betrachtung kommen indes Zweifel auf, ob der konzeptionelle Ansatz - Vorrang der internen Teilung - die Probleme des Versorgungsausgleichs in seiner geltenden Fassung überzeugend löst.
Die aus der Praxis vorgebrachte Kritik ${ }^{2}$ hat bislang nicht zu Änderungen oder Nachbesserungen des Entwurfs geführt; ob sich dieses im laufenden Gesetzgebungsverfahren ändern wird, bleibt abzuwarten.

\section{Die Zielsetzung: Interne Teilung der Anrechte}

Nach geltendem Recht erfolgt der Ausgleich der Versorgungsanwartschaften - dies darf vorausgeschickt werden - nach Saldierung aller Anrechte. Derjenige Ehepartner, der die werthöheren Anwartschaften erworben hat, ist in Höhe des hälftigen Wertunterschiedes ausgleichsverpflichtet, wobei - von ei-

\footnotetext{
1 BGH FamRZ 01, 1695 = NJW 02, 296 ff.; BVerfG NJW o6, 2177ff.)

2 (Rehme FamRZ 08, 738 ff. zu den verfassungsrechtlichen Bedenken; Born NJW 08, 2289 ff. zum „niedrigen Gerechtigkeitsniveau“.
} 
nigen Ausnahmen abgesehen - der Ausgleich allein über den gesetzlichen Versorgungsträger vorzunehmen ist. ${ }^{3}$

Unproblematisch ist die Begründung oder Übertragung von Anrechten, wenn beide Ehepartner nur über Anwartschaften in der gesetzlichen Rentenversicherung verfügen. Sobald Lebensversicherungen auf Rentenbasis oder eine betriebliche Altersversorgung hinzukommen, ist eine Bewertung der Anrechte vorzunehmen, um sie - zum Zwecke der Saldierung - vergleichbar zu machen.

Dies hat in der Vergangenheit wiederholt zu höchstrichterlichen Entscheidungen mit Blick auf die Beantwortung der Frage nach der dynamischen oder statischen Bewertung von Anrechten geführt. Für die Praxis nicht unbedeutend ist in jüngster Zeit auch die Entscheidung des Bundesgerichtshofs vom 14. November 2007 zur Ungleichbehandlung (sogenannter) rentenferner Versicherter $(=$ diejenigen, die am 1. Januar 2002 das 55. Lebensjahr noch nicht vollendet haben) in der Übergangsregelung der Versorgungsanstalt des Bundes und der Länder (VBL).

Solange die Satzungsbestimmungen der VBL nicht neu gefasst sind, werden die anhängigen Verfahren in der Regel abgetrennt und ausgesetzt. Damit verbunden sind zwangsläufig weitere „Restelisten“ (unabhängig von den ausgesetzten Verfahren gemäß $\mathbb{2}$ VAÜG), d.h. die Verfahren geraten unter dem Motto „Hauptsache geschieden“ in Vergessenheit, was häufig zu Lasten des ausgleichsberechtigten Ehepartners (= Ehefrau) geht.

Das Reformvorhaben besticht dagegen durch seine (vordergründig) vereinfachte Struktur, wonach die erworbenen Anrechte grundsätzlich der internen Teilung unterfallen ${ }^{4}$ und nur ausnahmsweise eine externe Teilung in Betracht kommt ${ }^{5}$. Interne Teilung bedeutet, dass der ausgleichsberechtigte Ehegatte in Höhe des Ausgleichswertes eigene Anwartschaften und Rechte gegenüber dem Versorgungsträger des anderen Ehegatten erwirbt, während eine externe Teilung nur durchzuführen ist, wenn der Versorgungsträger des pflichtigen Ehegatten mit dem berechtigten Ehegatten eine entsprechende Vereinbarung trifft oder der Versorgungsträger der ausgleichspflichtigen Person eine solche Teilung verlangt. ${ }^{6}$

Im Rahmen dieser Abhandlung soll ein wesentlicher Aspekt des Regelfalls (interne Teilung) genauer untersucht werden, nämlich der Ausgleich der Anrechte in der 2. Säule (= betriebliche Altersversorgung).

Die interne Teilung der Anrechte aus der betrieblichen Altersversorgung kann nämlich zu Ergebnissen führen, die mit dem Gedanken der „ge- rechten Teilhabe" der Eheleute an den in der Ehe erworbenen Anwartschaften nur schwer in Übereinstimmung zu bringen ist.

So haben die Versorgungswerke großer (privater) Unternehmen, wie z.B. Vertreter der Siemens AG oder der VW AG, anlässlich von Vortragsveranstaltungen eindringlich auf die Bedeutung und finanziellen Folgen der internen Teilung für den ausgleichsberechtigten Ehepartner (in der Regel die Ehefrau) aufmerksam gemacht.

Denn die betrieblichen Versorgungszusagen sind nach Inhalt und Umfang vielschichtig. So gibt es Anrechte, die leistungsbezogen sind (z.B. als Entgelt für ein Patent oder eine innovative Idee), die auf die Zugehörigkeit zum Betrieb abstellen und schließlich solche, die allein gehaltsbezogen sind. Nach dem Willen des Gesetzgebers sind alle Anwartschaften intern zu teilen.

Nach Auffassung der Versorgungsträger ist - bei der in Aussicht genommenen Regelung - für den ausgleichsberechtigten Ehepartner in „jeder Stufe“ eine versicherungsmathematische Neubewertung des Anrechts vorzunehmen, wobei die anfallenden Kosten - über deren Höhe man sich ausschweigt mit den Anrechten beider (oder eines) Ehepartners verrechnet werden können. ${ }^{7}$ Dass von dieser Regelung Gebrauch gemacht werden wird, unterliegt angesichts des erheblichen Verwaltungsaufwands keinen ernsthaften Zweifeln.

Um nicht über eine „Zweit- oder gar Drittehe“ des Mitarbeiters oder der Mitarbeiterin (eher selten) eine Vielzahl zusätzlicher Versorgungsberechtigter zu „erhalten“, wird daher auf Firmenebene in Erwägung gezogen, grundsätzlich nach $\mathbb{S} 47$ VAStrRefG vorzugehen und nur den korrespondierenden Kapitalwert mitzuteilen. Ob damit allerdings für die in der Regel ausgleichsberechtigte Ehefrau ein „Vorteil“ verbunden ist, darf kritisch hinterfragt werden. Hinzu kommt, dass kleinere Unternehmen sich überlegen werden, die betriebliche Zusatzversorgung abzubauen, um nicht nur den Verwaltungsaufwand nebst Kosten zu dezimieren, sondern sich auch „schwierige“ Berechnungen zu „ersparen“.

\section{Abänderbarkeit und Fehlerkorrektur}

Die Abänderbarkeit von Entscheidungen - heute in \10a VAHRG geregelt - wird künftig nicht mehr uneingeschränkt gegeben sein. Da der Entwurf von einem zeitgleichen Inkrafttreten des FamFG-E aus-

\footnotetext{
$3 \S \S 1587 a$ Abs.1, 1587b BGB.

4 $\oint 10 \mathrm{ff}$. VAStrRefG.

$5 \$ \$ 14 \mathrm{ff}$. VAStrRefG.

$6 \S 14$ Abs. 2 Nr. 1, 2 VAStrRefG.

$7 \S 13$ VAStrRefG.
}

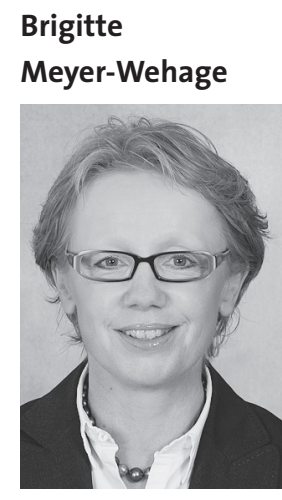

Mitglied der Kommission Zivil-, Familienund Erbrecht, Recht anderer Lebensgemeinschaften im djb, Richterin am Amtsgericht, Oldenburg 
geht, ist die Zulässigkeit und Durchführung einer Abänderung des Wertausgleichs bei der Scheidung in den $\mathbb{S} \mathbb{S} 225$ und 226 FamFG-E geregelt.

Danach findet eine Überprüfung nur statt, soweit die Rechte im Sinne des $\mathbb{3} 32$ VAStrRefG betroffen sind. Die Wertänderung muss zudem wesentlich sein, wobei nach Maßgabe der Entwurfsbegründung $\mathbb{S} 323 \mathrm{ZPO}^{8}$ heranzuziehen ist.

In diesem Zusammenhang darf jedoch nicht unerwähnt bleiben, dass eine „Fehlerkorrektur“ der Anrechte aus der 2. und 3. Säule ${ }^{9}$ nicht mehr möglich ist.

Die dagegen vorgebrachte Kritik ${ }^{10}$ ist durchaus berechtigt. Denn der Grundsatz der „gerechten Halbteilung “ wird (erneut) in Frage gestellt (unabhängig von der Verfassungsmäßigkeit der Regelung).

Ein weiterer (Kritik-)Punkt ist die Anpassung wegen Unterhalts. ${ }^{11}$ Die Vorschriften entsprechen dem $\mathbb{S}$ VAHRG mit dem - entscheidenden - Unterschied, dass die Korrektur nicht mehr durch den Leistungsträger des Verpflichteten erfolgt, sondern durch das Familiengericht ${ }^{12}$. Die sich abzeichnende Mehrbelastung der Gerichte ist bereits Gegenstand der Diskussion. ${ }^{13}$

\section{Ausblick}

Das (gesetzgeberische) Vorhaben einer umfassenden Reform des Versorgungsausgleichs ist - im Ergebnis - zu begrüßen. Denn die Alternative besteht allein in der Beibehaltung des bisherigen Systems mit versicherungsmathematischer „Nachberechnung “ (Modell Bergner).

Wünschenswert wäre jedoch eine Nachbesserung des Entwurfs aufgrund der im Detail geäußerten Vorbehalte gegenüber Einzelnormen des Entwurfs. Dies gilt auch für den Ausgleich der Anrechte aus der Beamtenversorgung. Denn die (nunmehr) für die Landesbediensteten zuständigen Bundesländer haben gegenüber dem Grundsatz der internen Teilung verfassungsrechtliche Bedenken (Grundsatz der Alimentation) erhoben, die durch $\$ 16$ VAStrRefG nicht als ausgeräumt angesehen werden. Um hier einen „Flickenteppich“ zu vermeiden, sollte (rechtzeitig) über eine einvernehmliche Lösung nachgedacht werden.

\footnotetext{
$8 \S 238$ FamFG-E.

9 Betriebliche Altersversorgung, Lebensversicherung auf Rentenbasis.

10 Born a.a.O. S. 2292 unter Hinweis auf Rehme FamRZ 08, 738 ff.

$11 \S \S 33,34$ VAStrRefG.

§ 34 Abs. 1 VAStrRefG.

Born a.a.O. S. 2293
}

\section{Das Kind im internationalen Familienrecht - ein kurzer Überblick}

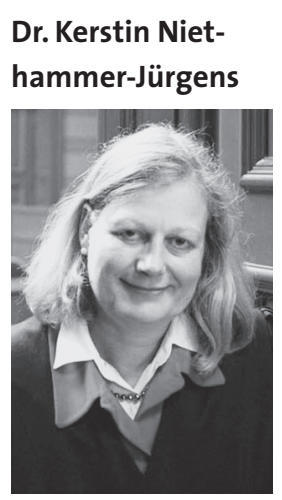

Mitglied der Kommission Zivil-, Familienund Erbrecht, Recht anderer Lebensgemeinschaften des djb, Rechtsanwältin und Fachanwältin für Familienrecht, Potsdam
Die anwaltliche familienrechtliche Beratung sieht sich einer zunehmenden Binationalität der Mandantschaft sowie einer wachsenden Zahl von Familien gegenüber, in denen eines oder mehrere Mitglieder nicht die deutsche Staatsangehörigkeit besitzen oder nicht in Deutschland leben. Ca. 700.000 deutsche Staatsangehörige haben einen ausländischen Ehepartner, allein an jeder sechsten in Deutschland geschlossenen Ehe ist ein nicht deutscher Partner beteiligt, wobei nur 2,7 Prozent dieser Eheschließungen ausschließlich unter Ausländern erfolgen. 2,5 Millionen minderjährige Kinder leben darüber hinaus in Deutschland, bei denen zumindest ein Elternteil nicht die deutsche Staatsangehörigkeit besitzt.

Nachdem die Ehen mit ausschließlicher Ausländerbeteiligung sowie die gemischt nationalen Ehen in ihrer Scheidungshäufigkeit den „rein deutschen “ Ehen in nichts nachstehen, liegt auch hier die Scheidungsrate bei knapp 45 Prozent.

Die Interessen der Kinder, die in einen Scheidungs- oder Trennungsprozess mit Auslandsbezug involviert sind, müssen in der innerfamiliären Auseinandersetzung besonders geschützt werden. Hierzu steht ein Normgefüge zur Verfügung, das insbesondere dem in Art. 8 der Europäischen Menschenrechtskonvention und Art. 6 GG festgeschriebenen
Schutz der Familie dient. Die internationalen Übereinkommen und EU-Verordnungen unterliegen damit der Anforderung an jede familiengerichtliche Entscheidung, diese zum Wohl und im Sinne des Kindes zu treffen. Dasjenige, was wir im deutschen Recht mit Kindeswohl umschreiben, durchzieht die Übereinkommen und Verordnungen, wobei allerdings die Ausgangs- und Schwerpunkte der insoweit einschlägigen Normen unterschiedlich und dabei ausgerichtet sind am Ziel der jeweiligen Norm.

\section{Brüssel Ila-VO (Eheverordnung)}

Soweit es um den Schutz des Kindes in Umgangsund Sorgerechtsverfahren geht, ist für Mitgliedstaaten der Europäischen Gemeinschaft (EU) mit Ausnahme Dänemarks seit dem 1. März 2005 auch für die neuen Mitgliedstaaten der EG nunmehr einschlägig die Eheverordnung ${ }^{1}$ (EheVO, auch Brüssel IIaVO genannt).

Die EheVO, die ihre Vorgängerverordnung bereits nach drei Jahren abgelöst hat, hat die Beschränkung

\footnotetext{
1 Verordnung (EG) Nr. 2201/2003 des Rates über die Zuständigkeit und die Anerkennung und Vollstreckung von Entscheidungen in Ehesachen und in Verfahren betreffend die elterliche Verantwortung und zur Aufhebung der Vorgängerverordnung (EG Nr. 1347/2000).
} 